Notre Dame Journal of Formal Logic

Volume 37, Number 1, Winter 1996

\title{
Elementary Propositions and Independence
}

\author{
JOHN L. BELL and WILLIAM DEMOPOULOS
}

\begin{abstract}
This paper is concerned with Wittgenstein's early doctrine of the independence of elementary propositions. Using the notion of a free generator for a logical calculus - a concept we claim was anticipated by Wittgensteinwe show precisely why certain difficulties associated with his doctrine cannot be overcome. We then show that Russell's version of logical atomism-with independent particulars instead of elementary propositions-avoids the same difficulties.
\end{abstract}

We intend to discuss a basic notion of logical atomism: that of independence. Elementary propositions are clearly central to Wittgenstein's atomism; their characterization rests on the doctrine of their independence. And a central tenet of Russell's atomism is that the world is decomposable into independent components. Our aim is to elucidate these two uses of independence and the conceptions of elementary proposition to which they give rise.

1 Elementary propositions as free generators One of the central and most problematic concepts in the Tractatus is that of 'elementarsatz' or elementary proposition. By this is meant a proposition in some sense not further analyzable into simpler propositions. Of this concept it is asserted in the Tractatus:

5.3 Every proposition is the result of truth operations on elementary propositions. ${ }^{1}$

That is, elementary propositions are to be regarded as the ultimate propositional constituents or generators of propositions: in this respect elementary propositions are propositional atoms, or atomic propositions. We also find the following further assertions concerning elementary propositions:

4.211 It is a sign of a proposition's being elementary that there can be no elementary proposition contradicting it.

5.134 One elementary proposition cannot be deduced from another.

Received August 17, 1995; revised February 19, 1996 
We may take these as asserting that elementary propositions are (logically) independent of one another. Now on what grounds can it be claimed that atomic propositions, in the sense of generators, satisfy this independence condition? To clarify this question, let us examine some familiar logical systems.

Consider first the propositional calculus. Here propositions are built up by applying the logical operators to an initial stock of proposition letters which are in another, completely natural sense, atomic propositions, since they are of minimal length. They are also independent in the strong sense that, for any finite set $\left\{P_{1}, \ldots, P_{n}\right\}$ of them, no conjunction of the form $X_{1} \& \cdots \& X_{n}$ is ever inconsistent, where each $X_{i}$ is either $P_{i}$ or $\neg P_{i}$.

This situation has a more general algebraic description. If, following the suggestion of 5.141, we identify (provably) equivalent propositions of the propositional calculus, we obtain a Boolean algebra - the Lindenbaum-Tarski algebra associated with the propositional calculus. A subset $X$ of a Boolean algebra $B$ is said to be free, and its elements independent, if for any finite subset $\left\{x_{1}, \ldots, x_{n}\right\}$ of $X$, we have $y_{1} \cap \cdots \cap y_{n} \neq 0$ where each $y_{i}$ is either $x_{i}$ or $x_{i}^{*}$ (= the Boolean complement of $x_{i}$ ). $X$ is said to generate $B$ if $B$ is the least subalgebra of $B$ containing $X$, in other words, if every element of $B$ can be expressed in the form $y_{1} \cup \cdots \cup y_{n}$, where each $y_{i}$ is of the form $z_{1} \cap \cdots \cap z_{m}$ with $z_{i} \in X$ or $z_{i}^{*} \in X .^{2}$ Finally $B$ is said to be freely generated if it has a free set of generators.

Now the Boolean algebra PROP associated with the propositional calculus is freely generated, and the propositional letters constitute a free set of generators. Thus, the propositional calculus provides a perfectly good, if restricted, model of Wittgenstein's scheme of elementary propositions, one that well illustrates his notion of elementarity, and the two notions of atomicity, introduced above. But Wittgenstein of course worked with a much richer language than that underlying the propositional calculus: in particular he required that universal and existential assertions be formulable. It is natural, therefore, to extend our discussion to the predicate calculus.

We first consider the pure predicate calculus PC. We assume that the underlying language contains a set $C$ of infinitely many constant symbols. Let PRED be the Boolean algebra obtained by identifying equivalent sentences in PC. PRED has a certain additional structure obtained by noting that quantified sentences are the suprema and infima of certain subsets, viz.,

$$
\begin{aligned}
& \exists x \varphi(x)=\sup \{\varphi(c): c \in C\} \\
& \forall x \varphi(x)=\inf \{\varphi(c): c \in C\} .
\end{aligned}
$$

For this reason we shall call PRED a quantifier algebra. ${ }^{3}$ This is, of course, analogous to Wittgenstein's discussion of quantification. Wittgenstein's account differs from the one we have given in terms of the notion of a quantifier algebra only in the choice of truth-function with respect to which he defines the quantifiers ${ }^{4}$; Wittgenstein uses a generalization of the Sheffer stroke rather than suprema and infima.

Now it can be shown that the set of atomic sentences (i.e., the set of corresponding equivalence classes) is free in PRED and that it also generates it as a quantifier algebra. (For a proof of this assertion, see Rasiowa and Sikorski [12], Chapter VIII, 24.1.) Thus, in this extended sense, PRED is freely generated by the set of atomic sen- 
tences, so that we obtain a satisfactory model of Wittgenstein's scheme, with atomic sentences again playing the role of elementary propositions (generators).

It has often been noted that Wittgenstein required of his elementary propositions that they be logically independent of one another. It has also been observed that elementary propositions constitute the atomic constituents of more complicated propositions. What appears to have been missed, so far as we can determine, is the significance of the combination of these two ideas, namely, that together they constitute an anticipation of the notion of a free generator. ${ }^{5}$ Thus, from our point of view it is not hard to see why Wittgenstein found his characterization of elementary propositions an attractive one; we can also give a complete answer to a question posed by David Pears:

"It is a sign of a proposition's being elementary that there can be no elementary proposition contradicting it." (Tractatus, 4.211) Why did Wittgenstein require the elementary propositions of the Tractatus to pass this difficult test? (10], p. 74)

On our account, Wittgenstein had, in effect, hit upon the idea of a free generator and had correctly noted, in the applications he made of the notion to the propositional calculus and to the pure predicate calculus, its connection with independence and with the notion of atomicity which we associate with minimal size; he also perceived its central role in these two logical systems. It is often remarked that the chief mathematical contribution of the Tractatus was Wittgenstein's presentation of the method of truth-tables. It seems to us that the articulation of the notion of a free generator together with the recognition of its centrality in the systems PROP and PRED was a contribution of no less importance.

2 Free generators and the Grundgedanke of the Tractatus Wittgenstein's "Grundgedanke" or fundamental thought is presented at 4.0312:

My fundamental thought is that the "logical constants" do not represent. That the logic of the facts cannot be represented.

Negation is the logical constant with respect to which the 'fundamental thought' is most fully elaborated in the Tractatus. In order to better see Wittgenstein's point in connection with negation, Ramsey ([11, pp. 146f) suggested that we should imagine the negation of an elementary proposition to be symbolically represented by inverting the sentence expressing it. His idea appears to have been that this would remove the temptation to think that in $\neg p$ the negation sign introduces an additional representational element not already present in $p$, a point made explicitly-or, at least, as explicitly as any point is ever made in the Tractatus - at 4.0621 (paragraph 3):

The propositions $p$ and $\neg p$ have opposite sense, but there corresponds to them one and the same reality.

In particular this would remove any basis for supposing that there must be negative facts in anything like the sense in which Russell appears to have supposed there to be negative facts, namely, as states of affairs existing alongside atomic facts. ${ }^{6}$

It seems to us that Ramsey's observation as well as other aspects of the fundamental thought are naturally captured by our account of elementary propositions as 
free generators. Let $E$ be the set of elementary propositions and $B$ the Boolean algebra freely generated by $E$, so that $B$ is the set of all propositions. We then have an injection $i: E \rightarrow B$ such that $i[E]$ is a free set of generators of $B$. We usually identify $p$ with $i(p)$ for $p \in E$. But this can cause problems since the map $i$ is not uniquely determined by $E$. For example, suppose given any such $i$, call it $i_{0}$, and any subset $X$ of $E$; define $i_{x}: E \rightarrow B$ by $i_{x}(p)=i_{0}(p)$ for $p \in X$, and $i_{x}(p)=i_{0}(p)^{*}$ for $p \in E-X$. Then $i_{x}: E \rightarrow B$ is an injection and $i_{x}[E]$ is also a free set of generators of B. ${ }^{7}$ Once elementary propositions are seen to be the atomic generators of all other propositions - once it is seen, in other words, that all propositions are truth functions of the elementary propositions-it remains to be observed only that the fundamental thought holds of negation, since the latitude allowed by $i$ extends only to the possibility of assigning $i(p)^{*}$ to an elementary proposition $p$.

If the injection of $E$ into $B$ is given by $i_{X}$, we may think of the elementary propositions in $X$ as being "given positively," and those not in $X$, "given negatively." But since $X$ is arbitrary, so is the positive/negative distinction. That is, the members of $E$ are "without orientation" (neither positive nor negative). They acquire their orientation only after insertion into $B$. Wittgenstein's pre-Tractarian notion that propositions are bipolar thus corresponds to the fact that, for $p \in E, i_{0}(p)$ as a free generator, could equally well be replaced by $i_{0}(p)^{*}: p$ itself does not determine which one we choose. $^{8}$

In the Tractatus, elementary propositions both picture reality and constitute the atomic generators of all other propositions. In our scheme, the distinction between these two functions is represented by the distinction between $p$ and $i(p)$. By 4.0621(3) (quoted earlier), the reality corresponding to $p$, if $p$ is true, is independent of its insertion into $B$ by the injection $i$. We have, therefore, to distinguish between $p$ as picture-something which does not depend on whether its image is given by $i$ or by $i_{x}$, and indeed, does not depend on $p$ 's insertion in $B$ at all —and $p$ 's role as a propositional generator of $B$; the latter does, of course, depend on $p$ 's insertion into $B$.

3 Independence lost Now it is well known that in [22] Wittgenstein came to question the doctrine of elementary propositions just outlined. What was the difficulty that caused him to do so? Consider the atomic sentences ' $G a$,' ' $R a$.' These are independent (free generators) in PRED. But if we now interpret ' $G$ ' as green, and ' $R$ ' as red, and ' $a$ ' as a speck in Ludwig's visual field, we turn them into contraries. As we see it, the difficulty is that Wittgenstein saw his account of elementary propositions not merely as a theory of 'empty forms,' but as one which would encompass at least one application. But in general, any specific application will have the effect of obliterating the independence of elementary propositions. Notice that the critical point was not the incompatibility of ' $a$ is red' and ' $a$ is green'-something already observed in the Tractatus (6.3751) — but the recognition that however the scheme of elementary propositions is applied, atomic propositions may no longer be independent, and so, a fortiori, no longer elementary. This is why, in Pears's phrase, independence is a "difficult test" of elementarity.

Wittgenstein's reference (at 4.04, quoted below) to Hertz's discussion of dynamical models (in [6], §§418-28), suggests that he saw his theory of elementary proposi- 
tions as a generalization of Hertz's account of "any possible mechanical description" (a point that is noted in Griffin [5], p. 5). The central idea which Wittgenstein took from Hertz's discussion of dynamical models was Hertz's notion that a dynamical model must have the right "mathematical multiplicity." A stumbling block to a correct interpretation of Wittgentein's appropriation of Hertz's concept of mathematical multiplicity is the juxtaposition of the two paragraphs which together make up 4.04:

In a proposition there must be exactly as many distinguishable parts as in the situation that it represents.

The two must possess the same logical (mathematical) multiplicity. (Compare Hertz's Mechanics on dynamical model.)

The first paragraph has misled commentators into supposing that Wittgenstein's (and Hertz's) use of mathematical multiplicity and dynamical models is captured by the notions of one-one correspondence and isomorphic image, with Wittgenstein (presumably following Hertz) adapting these notions to his conception of the proposition as picture. The idea, on this interpretation, is that sameness of mathematical multiplicity (which is identified with the existence of a one-one correspondence) is what is required if the names occurring in propositional signs are to be capable of "going proxy" for objects. ${ }^{9} 4.04$, however, consists of two paragraphs, and it is by no means evident that the second paragraph is merely a repetition, in other terminology, of the first. And indeed, an examination of 6 shows that the notion of mathematical multiplicity plays an altogether different and far subtler role, both in Hertz's discussion and in Wittgenstein's deployment of it. For Hertz, the requirement that a model have the right mathematical multiplicity meant that in an analysis of motion it is necessary to isolate the degrees of freedom characteristic of the system under study. In the simplest case - a single particle in free space — each of the three dimensions along which the particle's position can vary constitutes a "degree of freedom," and each component degree of freedom can be set independently of any other. ${ }^{10}$ This is the idea which is recalled when, just prior to his presentation of his general theory of propositions at $\S \S 5.5 \mathrm{ff}$, Wittgenstein tells us that such a theory requires

that we should construct a system of signs with a particular number of dimensions - with a particular mathematical multiplicity. (5.475)

Wittgenstein's notion of elementary proposition thus extended Hertz's notion of mathematical multiplicity or independence to the propositional generators of pure logic - the general form of any possible description. However, Wittgenstein failed to give any indication of how to recover the particular forms of description which underlie applications of this abstract framework. Obviously, the characterization of elementary propositions was not supposed to be dependent upon the features peculiar to its physical applications; nevertheless the account was supposed to encompass any such application. In the process of characterizing elementary propositions, Wittgenstein articulated a theoretically important mathematical concept- that of a free generator; however the goal of encompassing applications of his theory of elementary propositions eluded him. If, as Dummett [3] has persuasively argued, the principal achievement of Frege's theories of the cardinal and real numbers consisted in their ability to account for our applications of the numbers, then it is somewhat ironic that 
the fatal defect of Wittgenstein's theory of propositions should consist in its inability to provide for any application of the notion of an elementary proposition.

Formally, the situation may be described as follows. Let $T$ be any (consistent) theory in PC. We obtain a new Boolean algebra $\operatorname{PRED}_{T}$ by identifying sentences when they are provably equivalent in $T: \operatorname{PRED}_{T}$ is a quotient of PRED. Now, not only will the atomic sentences fail to be independent, and so, not a free set of generators in $\operatorname{PRED}_{T}$, the latter is, in general, not freely generated at all. Although atomic sentences are independent relative to the ordering defined by

$$
[\varphi] \leq[\psi], \text { if } \vdash \varphi \rightarrow \psi
$$

they may be dependent relative to the ordering

$$
[\varphi] \leq_{T}[\psi],
$$

given by

$$
\vdash_{T} \varphi \rightarrow \psi
$$

The issue which has attracted the attention of commentators is whether the ordering $\leq_{T}$ adequately captures the nature of the dependence of $[\varphi]$ and $[\psi]$. This is easily illustrated in terms of our earlier example: it happens that

$$
[R a] \leq_{T}[G a]^{*}
$$

so that $[R a]$ and $[G a]$ are dependent, since $T$ contains the axiom

$$
\forall x[R(x) \rightarrow \neg G(x)],
$$

which we may suppose governs what at 6.3751 Wittgenstein calls "the logical structure of colour." And indeed, the question raised in 22] is whether the nature of the incompatibility of red and green is adequately represented by the mere material truth of such an axiom, suggesting that the program implicit in the Tractatus's theory of elementary propositions required the discovery of analyses of apparently simple predicates like red and green, such that—under analysis-it would turn out that

$$
[R a] \leq[G a]^{*} .
$$

In other words, an analysis of ' $R a$ ' and ' $G a$ ' into properly elementary propositions should have the effect of allowing us to drop the reference to $T$ (or any other set of extralogical axioms). From this perspective, the doctrine of elementary propositions came to grief over the failure to discover such analyses, even for simple cases like red and green. But even if there were an analysis of red and green having the desired consequence, this would make no difference. Any analysis must be carried out in terms of some more basic family of predicates, and these predicates, whatever they happen to be, will sustain relations precisely like those found to hold between red and green - a fact that will call into question the soundness of supposing that the propositional atoms associated with the new predicates will prove to be independent. (For a related discussion, see Allaire [1].) The difficulty, therefore, is not the effect of accidental features of the applications we happen to have made of the predicate calculus, 
but is one which will arise in any material application of it. Initially, it might have been thought that the difficulty could be avoided by observing that it is merely independence relative to some $T$ which fails. However, once it is recognized that it isn't a matter of this or that analysis being right, but of every analysis apparently compromising the doctrine of the independence of elementary propositions, the question whether it is independence relative to $T$ or independence simpliciter which fails becomes overshadowed by the question whether there can be any plausible material application of the doctrine.

4 Independence regained: Russellian independence In any candidate application of Wittgenstein's theory of elementary propositions there will be numerous examples of propositional atoms which fail to meet the test of independence, and thus fail to be elementary in the sense of the theory. The question therefore arises of whether, aside from the fruitless search for "complete" analyses, there is any way the theory can accommodate such cases. Although there is an extensive secondary literature devoted to this issue, to our knowledge, no one has observed that there is a completely natural and easily motivated restriction on the requirement of independence which yields a salvageable form of the doctrine of elementary propositions. Moreover, it is in this form that the theory appears to have been advanced by Russell in his lectures of 1918, published as 16.

First, let us recall that the idea behind atomism as a physical hypothesis is that the world consists of physically separable and independent components. ${ }^{11}$ This suggests that logical atomism consists in the substitution of "logically separable and independent components" for "physically separable and independent components," an interpretation borne out by Russell's own explanations:

When I say logic is atomistic, I mean that I share the common-sense belief that there are many separate things (16, p. 178).

...the chief thesis that I have to maintain is the legitimacy of analysis, because if one goes into what I call Logical Atomism that means that one does believe the world can be analyzed into a number of separate things with relations and so forth...(16, p. 189).

And after introducing (in [16], Lecture II) the notion of a 'particular', which Russell compares with the classical notion of a substance, he explains that

...each particular has its being independently of any other and does not depend upon anything else for the logical possibility of its existence ([16, p. 203).

Thus, for Russell, it is, in the first instance, things or particulars that are independent, not facts or propositions-a remark which bears comparison with $\S 1$ of the Tractatus, especially:

1.2 The world divides into facts.

1.21 Each item can be the case or not the case while everything else remains the same.

But how are we to understand the proposal that one thing is logically independent of another? Isn't logical independence a relation among propositions, or perhaps, facts (states of affairs)? 
Now, in fact, it is not so difficult as it might first appear to make sense of the idea that things are independent. In quantum physics the assertion that two physical systems, $S$ and $S^{\prime}$, are independent is elliptical for the claim that if $Z$ is the phase space of $S$, and $Z^{\prime}$, the phase space of $S^{\prime}$, then the phase space of the combined system, $S+S^{\prime}$, is given by the tensor product $Z \otimes Z^{\prime}$ of the two phase spaces, and the state of the combined system is simply the product of the states of the components. In classical (particle) mechanics the independence would be represented by the claim that the phase space of the combined system is just the Cartesian product $\Omega \times \Omega$ of the phase spaces $\Omega$ and $\Omega^{\prime}$ of $S$ and $S^{\prime}$, respectively. Both representations make explicit the idea that the independence of $S$ and $S^{\prime}$ is based on the possibility of a representation of their respective magnitudes or properties under which they turn out to be algebraically independent in the sense explained earlier. This is the salient feature of both the classical and the quantum mechanical representations of $S+S^{\prime}$. So there is a sense in which the independence of things reduces to the independence of properties and propositions after all: roughly, independence of things consists in the independence of properties and propositions involving distinct things. In this sense, independence concerns the whole family of properties of $S$ and their relation to those of another system $S^{\prime}$, when nothing more is known of the two systems beyond the fact that they are two.

We should, at this point, emphasize a difference in the historical claims we are making regarding Russell and Wittgenstein. Although Russell's suggestions concerning the field of the relation of independence are, we contend, properly captured by the product constructions physicists employ in their representation of composite systems, we are not claiming that these mathematical constructions were in any way proposed by Russell as appropriate explications of his notion of independence. We do, however, see Wittgenstein as having explicitly articulated the central characteristics of the notion of a free generator, the basic mathematical idea underlying his conception of the independence of elementary propositions and atomic facts.

As we have presented it, atomism is a philosophical doctrine principally concerned with advancing the claim that there are independent facts (Wittgenstein) or the claim that there are independent things (Russell). Thus formulated, it is coincidental to either form of the doctrine whether the algebra of propositions associated with $S\left(S^{\prime}\right.$, or $\left.S+S^{\prime}\right)$ is, in the Boolean algebraic sense, atomic (i.e., contains minimal nonzero elements, technically called 'atoms'), since independence makes sense whether or not the algebra is atomic. Certainly, Wittgenstein's elementary propositions are not atomic in this sense, since atoms are not independent, but exclude one another. Moreover, if the initial stock of sentence letters is infinite, then PROP is, in the algebraic sense, atomless and a fortiori nonatomic. This is easily seen by noticing that if $\varphi$ is any noncontradictory proposition and $p$ a proposition letter not occurring in $\varphi$, then, in the Lindenbaum-Tarski algebra, the equivalence class of the element $\varphi \& p$ is strictly less than that of $\varphi$. (A similar remark holds for PRED. ${ }^{12}$.) As for the view we have attributed to Russell, there are of course independent propositions (as well as independent elementary propositions) in the representation of $S+S^{\prime}$ - this is the whole point of the tensor product and coproduct representations of (respectively) quantum and classical mechanical systems. ${ }^{13}$ But they are not atomic propositions in the sense of being atoms of the lattice of subspaces of $Z \otimes Z^{\prime}$ or of the Boolean algebra 
of subsets of $\Omega \times \Omega$. Again, if there are atoms, they are not independent but exclude one another. (In quantum mechanics, this is true only of "comeasurable" atoms.) But an atom associated with $S$ is independent of an atom associated with $S^{\prime}$ in their representation (as nonatoms) in the (quantum or classical mechanical) algebra of propositions associated with $S+S^{\prime} .{ }^{14}$

It is unlikely that Wittgenstein could have embraced Russellian independence, since it is so obviously motivated by Russell's preoccupation with British Idealism and the view that atomism is first and foremost a reaction to this tradition. Thus, for example, at 4.128 we read:

Logical forms are without number.

Hence there are no privileged numbers in logic, and hence there is no possibility of philosophical monism or pluralism, etc.

Indeed, juxtaposing Wittgenstein's doctrine of elementary propositions with Russell's conception of atomism suggests that Wittgenstein sought to free atomism from its connection with traditional metaphysical issues by displaying notions of independence and atomicity which do not require the truth of one or another "pseudoproposition" concerning the number of things. (Cf. also, 4.1272, paragraphs 4 and 5.) The point that we see Russell as having recognized is that in any application of the doctrine - in any material use of the notions of atomicity and independence - the significance of these notions will emerge from their applications to pluralities of physical (or whatever kinds of) systems. And in this he seems to have been right: Russell's notion of independence is preserved by the physicist's notion of 'logical' (i.e., phase) space, whereas Wittgenstein's is not. This is the intuition we see Wittgenstein to have lost sight of after his initial success in recovering the 'pure cases' of PROP and PRED.

Our contention that the core of the doctrine of elementary propositions is salvageable rests on the observation that although atomic sentences expressing properties of a single object may not be independent in $\operatorname{PRED}_{T}$, it is perfectly possible for quantifier-free sentences expressing properties of different objects to be independent. Moreover, individual atomic sentences can, under these conditions, still be contingent with respect to $T$. These desiderata are close to those Russell required of logical atomism.

To further clarify the point, let us introduce the concept of a Russellian theory. For each $c \in C$ let $\operatorname{Alg}(c)$ be the subalgebra of $\operatorname{PRED}_{T}$ consisting of quantifier-free sentences containing exactly the constant $c$. We shall call the theory $T$ Russellian if (1) for any atomic sentence $\sigma$, neither $\sigma$ nor $\neg \sigma$ is provable in $T$; (2) the $\operatorname{Alg}(c)$ for $c \in C$ are independent subalgebras of $\operatorname{PRED}_{T}$ : that is, for distinct $c_{1}, \ldots, c_{n}$, if $\sigma_{1} \in$ $\operatorname{Alg}\left(c_{1}\right), \ldots, \sigma_{n} \in \operatorname{Alg}\left(c_{n}\right)$ and $\sigma_{1} \& \ldots \& \sigma_{n}$ is inconsistent in $T$, then at least one $\sigma_{i}$ is refutable in $T$.

Notice that it would be unreasonable to expect condition (2) to be satisfied by sentences involving relations and quantifiers. For example, if $\sigma_{1}$ is the sentence $\forall y R c_{1} y$ and $\sigma_{2}$ is the sentence $\exists x R x c_{2}$, then clearly $\sigma_{1} \& \neg \sigma_{2}$, being a logical contradiction, is inconsistent in $T$, but there is no reason to suppose that either $\sigma_{1}$ or $\neg \sigma_{2}$ is refutable in $T$.

In 15, §§212-16, Russell discusses two views of relations which he calls the monadistic and monistic views. The monadistic view he attributes to Leibniz and 
Lotze, the monistic view, to Spinoza and Bradley. Both views share the common assumption that all propositions consist of a subject and (a monadic) predicate, and both require that any proposition which appears to attribute a relation to two or more individuals ("terms," as Russell says) must be given an analysis into subject-predicate form. The views differ in certain crucial respects on how this analysis is to be carried out. But for our purposes, what is interesting is the common framework they share. Following Russell's exposition, this common framework consists in the requirement that all propositions are of subject-predicate form, together with the corollary, which this is held to imply, that there are no external relations, relations which Russell characterizes as "implying no complexity in either of the related terms" (15], §214).

For the theories in question, the analysis of relational propositions containing asymmetric relations poses a special difficulty, since, in such cases, the analysis must not only show the existence of complexity in the related terms; it must also display an "intrinsic difference" between the terms of the relation:

we should be forced, in all cases of asymmetrical relations, to admit a specific difference between the related terms, although no analysis of either singly will reveal any relevant property which it possesses and the other lacks (15], §214).

Russell's discussion is subtle and complex, drawing as it does on a variety of metaphysical and logical concerns. To do it justice would require a separate paper. We do, however, think that our discussion of Russellian independence constitutes a development of one strand of this complex family of issues. It seems evident that the existence of external relations - the possibility, in other words, that two individuals can sustain a relation without this affecting their "individual complexity"-can be usefully compared to the thesis that there are independent things. When this is done, the absence of individual complexity simply becomes the independence of the characterizing algebras $\operatorname{Alg}\left(c_{1}\right)$ and $\operatorname{Alg}\left(c_{2}\right)$, and Russell's defense of external relations appears as an early anticipation of the stress he was later to place on independence.

Let $M$ be a theory formulated in the pure predicate calculus. We observe that in contrast with the theory of the Tractatus, it is perfectly possible for $M$ to be Russellian even though $\operatorname{PRED}_{M}$ is not freely generated: relative to $M$, atomic propositions concerning the same object fail to be independent, but atomic - even just quantifierfree-propositions concerning different objects can be independent. It can even be shown that atomic propositions generate $\operatorname{PRED}_{M}$. Thus, for Russellian theories, the doctrine of elementary propositions is salvageable, albeit in the weaker sense that only selected finite sets of elementary propositions are independent. To provide a foundation for these claims, we conclude with a very simple example of a Russellian theory. ${ }^{15}$

Let $W$ consist of the stars in the visible universe. In $W$ the only properties possessed by these individuals are mass and luminosity; the only relations to which they are subject are more massive than and brighter than. We will also suppose that mass and luminosity are quantized, so that measurements of these quantities always result in whole numbers. Thus we write $M_{i}(x)$ for " $x$ has mass $i$," $L_{i}(x)$ for " $x$ has luminosity $i$." So we assume that the language for $W$ contains, in addition to a name for each star, predicate symbols $M_{1}, M_{2}, \ldots ; L_{1}, L_{2}, \ldots$ and relation symbols $H$ (for more massive than) and $B$ (for brighter than). The theory $M$ of $W$ will express the mutual incompatibility of the $M_{i}$ and of the $L_{i}$, and the assumption that the $M_{i}$ and 
the $L_{i}$ are enumerated in increasing order of mass and luminosity, respectively. That is, $M$ has axioms:

$$
\begin{array}{rll}
\forall x\left[M_{i}(x) \rightarrow \neg M_{j}(x)\right] & \text { for } & i \neq j \\
\forall x\left[L_{i}(x) \rightarrow \neg L_{j}(x)\right] & \text { for } & i \neq j \\
\forall x, y\left[M_{i}(y) \& M_{j}(x) \rightarrow H(x, y)\right] & \text { for } & i<j \\
\forall x, y\left[L_{i}(y) \& L_{j}(x) \rightarrow B(x, y)\right] & \text { for } & i<j .
\end{array}
$$

It can be shown that $M$ is Russellian, and that $W$ is a "logically atomistic world." There seems to be nothing to prevent the theories of better approximations to the actual world — or even the theory of the actual world itself — from being Russellian. The fact that the standard classical and quantum mechanical representations of composite systems are Russellian lends further credibility to this observation.

Acknowledgments Support from the Natural Sciences and Engineering Research Council of Canada (J.L.B.) and the Social Sciences and Humanities Research Council of Canada (W.D.) is gratefully acknowledged. We wish to thank Jeffrey Bub, Robert Clifton, Michael Kremer and Mark Wilson for comments on an earlier draft.

\section{NOTES}

1. With one exception (4.0312, for which we use Ogden's translation) all quotations from the Tractatus are in the translation of Pears and McGuinness (Wittgenstein 211).

2. Here and elsewhere we ignore Wittgenstein's emphasis, in the Tractatus, on the Sheffer stroke, and consider generation with respect to the Boolean operations, $\cap, \cup$, *, and their propositional analogues, \&, $\vee \neg$.

3. Notice that when Wittgenstein says, "I dissociate the concept all from truth-functions" (5.521), he does not mean that he separates the quantifiers from truth-functions. As Mounce has observed, for Wittgenstein the notion of generality is not expressed by the quantifier but by our use of free variables. Cf. Mounce [9], pp. 68f.

4. On the peculiar centrality which the logicist tradition accorded the Sheffer stroke, see Goldfarb [4.

5. Everyone knows that although elementary propositions are supposed to be independent, there are difficulties in the way of combining independence and atomicity. But so far as we are aware, it has generally been missed that the combination is perfectly coherent, and indeed, mathematically fruitful, in the "pure" cases of PROP and PRED. The state of the secondary literature on this point is well represented by Rosenberg [14].

6. See Russell [16], Lecture II. Although a notion of a negative fact occurs in the Tractatus (at 2.06), it is defined - in contradistinction to Russell-as the nonexistence of an atomic fact.

7. A simple example, illustrating this situation, is afforded by the Boolean algebra $B_{16}$ of 16 elements associated with the propositional calculus over the proposition letters $p$ and $q$. This is the algebra whose elements are listed in the table given at 5.101. If we follow Wittgenstein's table, the elementary propositions $p$ and $q$ are identified with the truth functions [TTFF] and [TFTF], respectively. [TTTT] and [FFFF] are, respectively, the unit and the zero of the algebra, and the minimal nonzero elements of the algebra are [TFFF], [FTFF], [FFTF] and [FFFT]. In this example, $E=$ 
$\{p, q\}$; and $i[E]=\{[\mathrm{TTFF}]$, [TFTF $]\}$, which is a free set of generators of $\mathrm{B}_{16}$. But (for example) [FFTT], [FTFT] is also a free set of generators of $\mathrm{B}_{16}$ (as are the pairs $\{[\mathrm{TTFF}],[\mathrm{FTFT}]\} \&\{[\mathrm{FFTT}],[\mathrm{TFTF}]\})$. Each pair represents a different choice of injection of $p$ and $q$ into $\mathrm{B}_{16}$.

8. The positive/negative terminology can be somewhat confusing. Thus, Anscombe says of elementary propositions that they are "essentially positive," meaning by this that there are no negative facts in the sense in which Russell supposed there to be negative facts. As noted in the text, we take the rejection of Russellian negative facts to be the point of the observation of Ramsey which we are elucidating; given this understanding of the positive/negative distinction, we agree with Anscombe that elementary propositions - the propositions to which atomic facts correspond-are "essentially positive.' See Anscombe [2], pp. 31-33. For Wittgenstein's discussion of bipolarity, see Wittgenstein [20] §I. On Wittgenstein's use of bipolarity in connection with the fundamental thought, see McGuinness 8 .

9. Cf. for example, 14]. For an interesting discussion of mathematical multiplicity, generality and the picture theory of the Tractatus, see Kremer 7 .

10. Indeed, vector spaces are an exact analogue of the freely generated structures Wittgenstein considered, every vector space being freely generated by a set of basis vectors.

11. We ignore the question (which we grant is a real one) whether this formulation of the physical hypothesis of atomism is supported in its entirety by quantum mechanics. Our claims regarding quantum mechanics, which concern only the most general aspects of its representation of composite systems, do not depend on the resolution of this issue.

12. For a different view of Russell's and Wittgenstein's atomism, see Skyrms [18]. Skyrms argues that Russell and Wittgenstein differ over the coherence of supposing that the algebra of properties of a physical system is, or is not, atomless, with Russell allowing for this possibility, and Wittgenstein arguing against it. It is, however, unclear to us how Skyrms's discussion is related to our exposition, since, as noted in the text, PROP and PRED-though atomless-are perfect models of Wittgenstein's doctrine of elementary propositions.

13. For the coproduct of Boolean algebras, see Sikorski [17], §13, where the coproduct is called "the Boolean product." A closely analogous characterization of the tensor product of the lattice of subspaces of vector spaces is given in Stairs [19].

14. For further discussion of the concept of independence in elementary (i.e. nonrelativistic) quantum mechanics and in quantum field theory, see Rédei 13.

15. A simple but nontrivial example: for it is easily shown that the empty theory is Russellian.

\section{REFERENCES}

[1] Allaire, E. P., “Tractatus 6.3751,” Analysis, vol. 19 (1959), pp. 57-59.

[2] Anscombe, G. E. M., An Introduction to Wittgenstein's Tractatus, University of Pennsylvania Press, Philadelphia, 1971. 4

[3] Dummett, M., "What is mathematics about?," pp. 11-26 in Mathematics and Mind, edited by A. George, Oxford University Press, Oxford, 1994. MR 1373891 3

[4] Goldfarb, W., "Logic in the twenties: the nature of the quantifier," The Journal of Symbolic Logic, vol. 44 (1979), pp. 351-368.Zbl 0438.03001 MR 80j:03003 4 
[5] Griffin, James, Wittgenstein's Logical Atomism, Oxford University Press, Oxford, 1964. 3

[6] Hertz, H., The Principles of Mechanics, translated by D. E. Jones and J. T. Walley, Dover, New York, 1956.ZZbl 0074.38805|MR 17,1017f 3,3

[7] Kremer, M., "The multiplicity of general propositions," Noûs, vol. 26 (1992), pp. 409426. MR 1262540 4

[8] McGuinness, B., "The Grundgedanke of the Tractatus," pp. 49-61 in Understanding Wittgenstein, edited by G. Vesey, Macmillan, London, 1974.

[9] Mounce, H. O., Wittgenstein's Tractatus: An Introduction, University of Chicago Press, Chicago, 1981.

[10] Pears, D., "The logical independence of elementary propositions," pp. 74-84 in Perspectives on the Philosophy of Wittgenstein, edited by I. Block, Blackwell, Oxford, 1981.

[11] Ramsey, F. P., "Facts and propositions," pp. 138-155 in The Foundations of Mathematics and Other Logical Essays, edited by R. B. Braithwaithe, Littlefield Adams and Co., Paterson, 1960.

[12] Rasiowa, H., and R. Sikorski, The Mathematics of Metamathematics, Panstowe Wydawnictwo Naukowe, Warsaw, 1963. Zb1 0122.24311MR 29:1149 1

[13] Rédei, M. "Logical independence in quantum logic," Foundations of Physics, vol. 25 (1995), pp. 411-422. MR 96b:81015 4

[14] Rosenberg, J. F. “Wittgenstein's theory of language as picture," American Philosophical Quarterly, vol. 5 (1968), pp. 18-30. 4.4

[15] Russell, B., The Principles of Mathematics, Norton, New York, 1902. 4.4.4.4

[16] Russell, B., The Philosophy of Logical Atomism, in Logic and Knowledge: Essays 1901-1950, edited by R. Marsh, George Allen and Unwin, London, 1956. 4,4, 4, 4, 4. 4

[17] Sikorski, R., Boolean Algebras, Second edition, Springer-Verlag, Berlin, 1960. Zbl 0087.02503||MR 23:A3689 4

[18] Skyrms, B., "Logical atomism and combinatorial possibility," The Journal of Philosophy, vol. 90 (1993), pp. 219-232. 4

[19] Stairs, A., "On the logic of pairs of quantum systems," Synthese, vol. 56 (1983), pp. 4760. Zbl 0518.03028|MR 85e:81011 4

[20] Wittgenstein, L., "Notes on Logic (1913)," pp. 93-106 in Notebooks, 1914-1916, edited and translated by G. E. M. Anscombe, Basil Blackwell, Oxford, 1961. 4

[21] Wittgenstein, L., Tractatus Logico-Philosophicus, translated by D. Pears and B. McGuinness, Routledge \& Kegan Paul, London, 1961.

[22] Wittgenstein, L., "Some remarks on logical form," Proceedings of the Aristotelian Society, Supplementary Volume IX (1929), pp. 162-171. 3,3

Department of Philosophy

The University of Western Ontario

London, Ontario N6A $3 K 7$

Canada

email: ibell@iulian.uwo.ca

email: wgdemo@uwovax.uwo.ca 